\title{
Interactive comment on "Distinctions in source regions and formation mechanisms of secondary aerosol in Beijing from summer to winter" by Jing Duan et al.
}

Jing Duan et al.

rujin.huang@ieecas.cn

Received and published: 8 June 2019

This is a revised supplement to the manuscript. Please download the attached pdf.

Please also note the supplement to this comment:

https://www.atmos-chem-phys-discuss.net/acp-2019-16/acp-2019-16-AC3-

supplement.pdf 2019. 